PROCEEDINGS OF THE

AMERICAN MATHEMATICAL SOCIETY

Volume 91, Number 3, July 1984

\title{
RELATIONS FOR GROTHENDIECK GROUPS OF ARTIN ALGEBRAS
}

\author{
MAURICE AUSLANDER
}

\begin{abstract}
M. C. R. Butler has shown that if $\Lambda$ is an artin algebra of finite representation type, then the almost split sequences generate the relations for the Grothendieck group of $\Lambda$. This paper is primarily devoted to proving the converse of Butler's result, i.e. $\Lambda$ is of finite representation type if the almost split sequences generate the relations for the Grothendieck group of $\Lambda$.
\end{abstract}

Introduction. Throughout this note we assume $R$ is a commutative artin ring and $\Lambda$ is an $R$-algebra which is a finitely generated $R$-module. All $\Lambda$-modules are assumed to be finitely generated left $\Lambda$-modules. We denote the category of all such modules by $\bmod \Lambda$. Also we choose a fixed full subcategory ind $\Lambda$ of $\bmod \Lambda$ whose objects are a complete set of nonisomorphic indecomposable $\Lambda$-modules. Letting $K_{0}(\bmod \Lambda, 0)$ be the free abelian group with basis the isomorphism classes $[M]$ of $\Lambda$-modules $M$ modulo the subgroup generated by elements of the form $[A]+[C]-$ $[A \amalg C]$, we have the following well known facts:

(a) The set $\{[M] \mid M$ in ind $\Lambda\}$ is a free basis for $K_{0}(\bmod \Lambda, 0)$;

(b) $K_{0}(\bmod \Lambda, 0)$ modulo the subgroup generated by elements of the form $[A]+$ $[C]-[B]$, where there is an exact sequence $0 \rightarrow A \rightarrow B \rightarrow C \rightarrow 0$, is the Grothendieck group $K_{0}(\bmod \Lambda)$ of $\bmod \Lambda$.

Since the $[M]$ with $M$ in ind $\Lambda$ form a natural basis for the generators of $K_{0}$ $(\bmod \Lambda)$ it is reasonable to ask if there is a natural basis for the relations of $K_{0}(\bmod \Lambda)$, i.e. for $\operatorname{Ker} \phi$ where $\phi: K_{0}(\bmod \Lambda, 0) \rightarrow K_{0}(\bmod \Lambda)$ is the canonical epimorphism. In [5] M. C. R. Butler showed that if $\Lambda$ is of finite representation type (i.e. ind $\Lambda$ has only a finite number of objects) then $\operatorname{Ker} \phi$ is generated by the elements $[A]+[C]-[B]$ in $K_{0}(\bmod \Lambda, 0)$ such that there is a special kind of exact sequence $0 \rightarrow A \rightarrow B \rightarrow C \rightarrow 0$ called an almost split sequence. One of our aims in this note is to show that the generators given by Butler are linearly independent as well as proving the converse of Butler's theorem. The fact that we had obtained these results was announced by Butler in [5] but the proofs were never published.

As was the case with Butler's work, our proofs depended heavily on categorical arguments. Another of our purposes here is to show that these functorial arguments can be avoided by considering the bilinear form $\langle\rangle:, K_{0}(\bmod \Lambda, 0) \times K_{0}(\bmod \Lambda, 0) \rightarrow$ $\mathbf{Z}$ given by $\langle A, B\rangle=l_{R} \operatorname{Hom}(A, B)$ for all $A$ and $B$ in $\bmod \Lambda$ where $l_{R}$ means length as a $R$-module. While this form has been used implicitly in various situations, Benson and Parker (see [4]) were the first to consider it explicitly and develop its basic properties, some of which we include for the sake of completeness in the following section.

Received by the editors July 26, 1983 .

1980 Mathematics Subject Classification. Primary 16A46, 16A54, 16 A64.

(C) 1984 American Mathematical Society $0002-9939 / 84 \$ 1.00+\$ .25$ per page 
1. Basic properties of the bilinear form. The properties of the form of interest to us are derived from the existence and basic properties of almost split sequences. We start this section with a brief review of almost split sequences (see $[2, \mathbf{3}]$ for more details).

An exact sequence $0 \rightarrow A \rightarrow B \rightarrow C \rightarrow 0$ is called an almost split sequence if it has the following properties:

(a) $A$ and $C$ are indecomposable.

(b) For each $X$ in ind $\Lambda$ define the End $X^{\text {op }}$-module $S_{C}(X)$ by means of the exact sequence of End $X^{\mathrm{op}}$-modules

$$
0 \rightarrow(X, A) \rightarrow(X, B) \rightarrow(X, C) \rightarrow S_{C}(X) \rightarrow 0
$$

where $($,$) means \operatorname{Hom}_{\Lambda}($,$) . Then$

(i) $S_{C}(X)=0$ if $X \neq C$,

(ii) $S_{C}(X)$ is the unique simple End $C^{\text {op }}$-module if $X=C$.

(c) For each $X$ in ind $\Lambda$, define the End $X$-module $S^{A}(X)$ by means of the exact sequence of End $X$-modules $0 \rightarrow(C, X) \rightarrow(B, X) \rightarrow(A, X) \rightarrow S^{A}(X) \rightarrow 0$. Then

(i) $S^{A}(X)=0$ if $A \neq X$,

(ii) $S^{A}(X)$ is the unique simple End $A$-module when $X=A$.

We now associate with each $X$ in ind $\Lambda$ elements $r_{x}$ and $l_{x}$ in $K_{0}(\bmod \Lambda)$ which play a fundamental role in the rest of this note.

Let $C$ be in ind $\Lambda$. If $C$ is not projective, then there is a unique, up to isomorphism almost split sequence $0 \rightarrow A \rightarrow B \rightarrow C \rightarrow 0$ (see [2]). We then associate with $C$ the element $r_{C}=[A]+[C]-[B]$ in $K_{0}(\bmod \Lambda, 0)$. If $C$ is projective we let $r_{C}=[C]-[\underline{r} C]$ where $\underline{r}$ is the radical of $\Lambda$. The following properties of the elements $r_{C}$ will be used constantly in this note.

LEMMA 1.1. Let $C$ be in ind $\Lambda$.

(a) For each $X$ in ind $\Lambda$ we have $\left\langle X, r_{C}\right\rangle=0$ if $X \neq C$ and $r_{C}=l_{R}\left(S_{C}(X)\right) \neq 0$ if $X=C$;

(b) $\langle C, X\rangle /\left\langle C, r_{C}\right\rangle=l_{\text {End } C \text { op }}(C, X)$ for all $X$ in ind $\Lambda$.

PROOF. The proof is an easy consequence of (b) of the definition of almost split sequences given above and that $\underline{r} C$ is the unique maximal submodule of an indecomposable projective module $C$ (see [4, Theorem 3.4]).

We now define $l_{A}$ for each $A$ in ind $\Lambda$. If $A$ is not injective, there is a unique up to isomorphism almost split sequence $0 \rightarrow A \rightarrow B \rightarrow C \rightarrow 0$. We then associate with $A$ the element $l_{A}=[A]+[C]-[B]$ in $K_{0}(\bmod \Lambda, 0)$. If $A$ is injective, then define $l_{A}=[A]-[A / \operatorname{Soc} A]$, where Soc $A$ is the socle of $A$. The following properties of the elements $l_{A}$ will be used constantly in this note.

LEMMA 1.2. Let $A$ be in ind $\Lambda$.

(i) For each $X$ in ind $\Lambda$ we have $\left\langle l_{A}, X\right\rangle=0$ if $A \neq X$ and $\left\langle l_{A}, X\right\rangle=l_{R} S^{A}(X)$ $\neq(0)$ if $X=A$.

(ii) $\langle X, C\rangle /\left\langle l_{C}, C\right\rangle=l_{\text {End } C}(X, C)$ for all $X$ in ind $\Lambda$.

PROOF. Dual of Lemma 1.1.

The main reason for introducing the elements $r_{C}$ and $l_{A}$ in $K_{0}(\bmod \Lambda, 0)$ is given in the following. 
Proposition 1.3. Let $x$ be an element in $K_{0}(\bmod \Lambda, 0)$. Then we have (a) $\left\langle x, r_{C}\right\rangle /\left\langle C, r_{C}\right\rangle$ is an integer for each $C$ in ind $\Lambda$ and

$$
x=\sum_{C \in \text { ind } \Lambda}\left(\left\langle x, r_{C}\right\rangle /\left\langle C, r_{C}\right\rangle\right)[C] .
$$

(b) $\left\langle l_{A}, x\right\rangle /\left\langle l_{A}, A\right\rangle$ is an integer for each $A$ in ind $\Lambda$ and

$$
x=\sum_{A \in \text { ind } \Lambda}\left(\left\langle l_{A}, x\right\rangle /\left\langle l_{A}, A\right\rangle\right)[A] .
$$

Proof. (a) Let $x=\sum_{C \in \text { ind } \Lambda} a_{C}[C]$. Then $\left\langle x, r_{X}\right\rangle=a_{X}\left\langle X, r_{X}\right\rangle$ for all $X$ in ind $\Lambda$ since $\left\langle C, r_{X}\right\rangle=0$ if $C \neq X$.

(b) Proven similarly.

As an immediate consequence of this proposition we have

COROLLARY 1.4. The following are equivalent for $x$ in $K_{0}(\bmod \Lambda, 0)$ :

(a) $x=0$;

(b) $\langle x, C\rangle=0$ for all $C$ in ind $\Lambda$;

(c) $\langle A, x\rangle=0$ for all $A$ in ind $\Lambda$.

The following criteria for modules to be isomorphic is an immediate consequence of this corollary. An earlier unpublished functorial proof now appears in [1].

COROLlaRY 1.5. The following are equivalent for $X$ and $Y$ in $\bmod \Lambda$.

(a) $X \cong Y$.

(b) $\langle X, C\rangle=\langle Y, C\rangle$ for all $C$ in ind $\Lambda$.

(c) $\langle A, X\rangle=\langle A, Y\rangle$ for all $A$ in ind $\Lambda$.

ProOF. Let $x=[X]-[Y]$ in Corollary 1.2.

We now apply Corollary 1.5 to prove the following "converse" of that corollary. ind $\Lambda$.

PROPOSITION 1.6. The following are equivalent for a full subcategory $\underline{\underline{C}}$ of

(a) $\underline{\underline{C}}=\operatorname{ind} \Lambda$.

(b) $\overline{\bar{X}}$ and $Y$ in $\bmod \Lambda$ are isomorphic if $\langle C, X\rangle=\langle C, Y\rangle$ for all $C$ in $\underline{\underline{C}}$.

(c) $X$ and $Y$ in $\bmod \Lambda$ are isomorphic if $\langle X, C\rangle=\langle Y, C\rangle$ for all $C$ in ind $\Lambda$.

Proof. That (a) implies (b) and (c) has already been shown.

(b) implies (a). Suppose $Z$ is in ind $\Lambda-\underline{C}$. Then $\left\langle C, r_{Z}\right\rangle=0$ for all $C$ in $\underline{C}$. If $Z$ is projective, then this implies that $\langle C, \underline{r} Z\rangle=\langle C, Z\rangle$ for all $C$ in $\underline{\underline{C}}$. Hence $\underline{\bar{r}} Z \cong Z$ which is a contradiction. If $Z$ is not projective and $r_{Z}=X-Y \overline{\bar{F}} Z$ then we have that $\langle C, X \amalg Z\rangle=\langle C, Y\rangle$ for all $C$ in $\underline{\underline{C}}$. Hence $X \amalg Z=Y$ which contradicts the fact that $0 \rightarrow X \rightarrow Y \rightarrow Z \rightarrow 0$ is an almost split sequence since in such a sequence $Z$ is not a summand of $Y$ (see [2, Proposition 3.3]). Hence ind $\Lambda-\underline{\underline{C}}=\emptyset$ which shows that (b) implies (a).

We end this section with the following easily verified but important result.

Proposition 1.7. Each of the sets of elements $\left\{r_{C}\right\}_{C \in \operatorname{ind} \Lambda}$ and $\left\{l_{C}\right\}_{C \in \text { ind } \Lambda}$ in $K_{0}(\bmod \Lambda, 0)$ is linearly independent.

Proof. This is an easy consequence of the fact that if $X$ and $C$ are in ind $\Lambda$, then $\left\langle X, r_{C}\right\rangle=0=\left\langle l_{C}, X\right\rangle$ if and only if $X \neq C$. 
2. Proof of main results. This section is devoted to applying the results in $\S 1$ to proving the results described in the introduction. We begin with some preliminary results which are of interest in their own right.

PROPOSITION 2.1. Suppose $\Lambda$ is of finite representation type. Then for each $x$ in $K_{0}(\bmod \Lambda, 0)$ we have

(a) $\langle C, x\rangle /\left\langle C, r_{C}\right\rangle$ is an integer for each $C$ in ind $\Lambda$ and

$$
x=\sum_{C \in \operatorname{ind} \Lambda}\left(\langle C, x\rangle /\left\langle C, r_{C}\right\rangle\right) r_{C} .
$$

(b) $\langle x, A\rangle /\left\langle l_{A}, A\right\rangle$ is an integer for each $A$ in ind $\Lambda$ and

$$
x=\sum_{A \in \operatorname{ind} \Lambda}\left(\langle x, A\rangle /\left\langle l_{A}, A\right\rangle\right) l_{A} .
$$

Hence $\left\{r_{C}\right\}_{C \in \operatorname{ind} \Lambda}$ and $\left\{l_{A}\right\}_{A \in \text { ind } \Lambda}$ are free bases for $K_{0}(\bmod \Lambda, 0)$.

Proof. (a) That $\langle C, x\rangle /\left\langle C, r_{C}\right\rangle$ is an integer for each $C$ in ind $\Lambda$ follows from the fact that $\langle C, X\rangle /\left\langle C, r_{C}\right\rangle=l_{\text {End } C \text { op }}(C, X)$ for each $X$ in ind $\Lambda$ (see Lemma 1.1). Therefore $y=\sum_{C \in \operatorname{ind} \Lambda}\left(\langle C, x\rangle /\left\langle C, r_{C}\right\rangle\right) r_{C}$ is an element of $K(\bmod \Lambda)$. Since $\langle C, x\rangle=\langle C, y\rangle$ for all $C$ in ind $\Lambda$, it follows by Corollary 1.4 that $x=y$ which proves (a). (b) Proven similarly to part (a).

The rest of the proposition is a trivial consequence of $(\mathrm{a})$ and $(\mathrm{b})$ and Proposition 1.7 .

We can now prove the following characterization of $\Lambda$ being of finite representation type.

THEOREM 2.2. The following statements are equivalent for $\Lambda$.

(a) $\Lambda$ is of finite representation type.

(b) $\left\{r_{C}\right\}_{C \in \text { ind } \Lambda}$ generates $K_{0}(\bmod \Lambda, 0)$.

(c) $\left\{l_{A}\right\}_{A \in \text { ind } \Lambda}$ generates $K_{0}(\bmod \Lambda, 0)$.

Proof. Since we have just proven that (a) implies (b) and (c), it only remains to show that (b) implies (a) and (c) implies (a).

(b) implies (a). Let $X$ be in ind $\Lambda$. Then $X=\sum_{C \in \text { ind } \Lambda} a_{X, C} r_{C}$ and so $\langle C, X\rangle=$ $a_{X, C}\left\langle C, r_{C}\right\rangle$ for each $C$ in ind $\Lambda$. Since only a finite number of $a_{X, C} \neq 0$, it follows that there are only a finite number of $C$ in ind $\Lambda$ such that $\langle C, X\rangle \neq 0$. This implies $\Lambda$ is of finite representation type since every $C$ in ind $\Lambda$ has a nonzero morphism to a simple $\Lambda$-module and there are only a finite number of simple modules in ind $\Lambda$.

(c) implies (a). Similar to (b) implies (a).

We now show how Theorem 2.2 gives the results promised in the introduction.

THEOREM 2.3. Let $\underline{P}$ be the full subcategory of ind $\Lambda$ consisting of the projective modules in ind $\Lambda$. Then $\left\{r_{C}\right\}_{C \in \operatorname{ind} \Lambda-\underline{P}}$ generates $\operatorname{Ker} \phi$ where $\phi: K_{0}(\bmod \Lambda, 0) \rightarrow$ $K_{0}(\bmod \Lambda)$ is the canonical surjection if and only if $\Lambda$ is of finite representation type. Further, if $\Lambda$ is of finite representation type, then $\left\{r_{C}\right\}_{C \in \operatorname{ind} \Lambda-\underline{\underline{P}}}$ is a basis for $\operatorname{Ker} \phi$.

Proof. First we observe that $\phi\left(r_{C}\right)=0$ if $C$ is not projective and $\phi\left(r_{C}\right)=$ $[C / \underline{r} C]$ if $C$ is projective. Hence if $\left\{r_{C}\right\}_{C \in \operatorname{ind} \Lambda-\underline{\underline{P}}}$ generates Ker $\phi$, then

$$
\left\{r_{C}\right\}_{C \in \operatorname{ind} \Lambda-\underline{\underline{P}}} \cup\left\{r_{P}\right\}_{P \in \underline{\underline{P}}}
$$


generates $K_{0}(\bmod \Lambda$,$) since \left\{\phi\left(r_{P}\right)\right\}_{P \in \underline{P}}=\{[P / \underline{r} \phi]\}_{P \in \underline{\underline{P}}}$ generates $K_{0}(\bmod \Lambda)$. It now follows from Theorem 2.2 that $\Lambda$ is of finite representation type.

Suppose now that $\Lambda$ is of finite representation type. Then by Theorem 2.2 we know that $\left\{r_{C}\right\}_{C \in \text { ind } \Lambda}$ is a basis for $K_{0}(\bmod \Lambda, 0)$ and so each element $x$ in $K_{0}(\bmod \Lambda, 0)$ can be written as $\sum_{C \in \underline{P}} a_{C} r_{C}+\sum_{P \in \underline{P}} a_{P} r_{P}$. Now $\phi(x)=0$ if and only if $\left.\sum_{P \in \underline{\underline{P}}} a_{P} \phi\left(r_{P}\right)=\sum_{P \in \underline{\underline{P}}} a_{P} \overline{\overline{[P}} / \underline{r} P\right]=0$. But $\sum_{P \in \underline{\underline{P}}} a_{P}[P / \underline{r} P]=0$ in $K_{0}(\bmod \Lambda)$ if and only if each $a_{P}=0$ since the family of elements $\{[P / r P]\}_{P \in \underline{P}}$ in $K_{0}(\bmod \Lambda)$ is a basis for $K_{0}(\bmod \Lambda)$. Hence if $x$ is in $\operatorname{Ker} \phi$, then $x=\sum_{C \notin \underline{P}} a_{C} r_{C}$ and so $\left\{r_{C}\right\}_{C \in \text { ind } \Lambda-\underline{P}}$ generates $\operatorname{Ker} \phi$. Clearly $\left\{r_{C}\right\}_{C \in \text { ind } \Lambda-\underline{P}}$ is linearly independent since by Proposition 1.7 we have that $\left\{r_{C}\right\}_{C \in \text { ind } \Lambda}$ is linearly independent.

\section{REFERENCES}

1. M. Auslander and I. Reiten, Modules determined by their composition factors, Illinois J. Math. (to appear).

2. __ Representation theory of artin algebras. III, Comm. Algebra 3 (1975), 239-294.

3. __ Representation theory of artin algebras. IV, Comm. Algebra 5 (1977), 443-518.

4. D. J. Benson and R. A. Parker, The Green ring of a finite group, Aarhus University, April, 1982.

5. M. C. R. Butler, Grothendieck groups and almost split sequences, Lecture Notes in Math., vol. 822, Springer-Verlag, Berlin and New York, 1981.

Department of Mathematics, Brandeis University, Waltham, MassachuSETTS 02254 